\title{
Metaanalysen als Königsweg für die Formulierung von Therapieleitlinien?
}

\author{
Manfred M. Fichter \\ Medizinisch-Psychosomatische Klinik Roseneck, Prien am Chiemsee, Deutschland
}

Als ich Student war, war die Appendektomie (Blinddarmentfernung) auch bei leichter Blinddarmreizung weit verbreitet, ohne dass die Sinnhaftigkeit dieses Vorgehens empirisch wirklich ausreichend untersucht worden war. Die Annahme, dass dies helfen müsse, wurde schlicht als richtig akzeptiert (Augenscheinvalidität) und erst Jahrzehnte später hinterfragt. Heute ist es allgemein akzeptiert, dass Therapieempfehlungen auf empirischer Evidenz zu basieren haben. Der Grundgedanke ist dabei, dass alle relevanten empirischen Untersuchungen gleichermaßen berücksichtigt werden. Die evidenzbasierte Medizin (besser: das evidenzbasierte Gesundheitswesen) soll sich auf die Schnittmenge aller Einzelevidenzen stützen und zu einer Gesamtschlussfolgerung zu einem bestimmten Thema kommen. Im Deutschen wird der Begriff «Evidenz» in der Regel im Sinne von intuitiver Erfahrung verwendet, während im Kontext von evidenzbasierter Medizin (EBM) darunter die Summe empirischen Wissens zu einem Sachverhalt verstanden wird. Ressourcen sollen möglichst auf der Basis der Ratio eingesetzt werden, wobei Ratio von den einen als «vernunftgeleitet», von den anderen als «einsparungsgeleitet» verstanden wird.

Auch als praktisch tätige Psychotherapeuten sind wir zunehmend von diesen relativ abstrakten Konzepten betroffen. Die Konzepte der EBM haben in den letzten Jahren auch stark auf die Konzeptualisierung von Psychotherapiestudien übergegriffen. Die Grundintention ist sicher begrüßenswert: Für jede Störung oder Erkrankung soll das Therapieverfahren zum Einsatz kommen, das sich in ausreichend vielen empirischen Untersuchungen als wirkungsvoll erwiesen hat. Die Therapie soll dem Patienten mit möglichst hoher Sicherheit möglichst gut helfen, seine Erkrankung zu bewältigen. Sowohl für den praktizierenden als auch für den forschenden Psychotherapeuten ist es ratsam und wichtig, sich mit Fragen der Leitlinienentwicklung und den Empfehlungen, die diese aussprechen, zu befassen.

Man kann zwei Arten systematischer Evidenzgewinnung unterscheiden: (1) Die kumulative Wertung empirischer Ein- zeluntersuchungen in Form von Übersichtsarbeiten und systematischen Reviews. (2) Die kumulative Wertung von empirischen Einzeluntersuchungen in Form von Metaanalysen; bei letzteren kommen statistische Verfahren (Berechnung von Effektstärken, Konfidenzintervallen und z.B. Log-odds-Ratios) zur Anwendung, die zu einer abschließenden kumulativen Wertung führen. Das hört sich gut an!

In den letzten Jahren wird in Deutschland zunehmend Metaanalysen der Vorrang vor systematischen Reviews gegeben. Evidenz aufgrund von Metaanalysen von mindestens drei randomisierten kontrollierten Studien (RCT) hat nach den Festlegungen der «Agency of Health Care Policy and Research» [Berger et al., 2004] die höchste Evidenzstufe (Ia). Danach folgen in absteigender Reihenfolge mindestens 1 RCT oder Metaanalysen von weniger als 3 RCTs (Ib), mindestens 1 kontrollierte nichtrandomisierte Studie mit methodisch hochwertigem Design (IIa), mindestens 1 quasi-experimentelle Studie mit methodisch hochwertigem Design (IIb), mindestens 1 nichtexperimentelle deskriptive Studie (Vergleichsstudie, Korrelationsstudie, Fallserien) (III) und Berichte/Empfehlungen von Expertenkomitees oder klinische Berichte anerkannter Autoritäten (IV). Dies ist ein Beispiel für eine Evidenzgraduierung. Verschiedene Gremien und Institutionen haben unterschiedliche Definitionen für Evidenzgraduierung. Das erschwert die Vergleichbarkeit und Kommunikation. Möller und Maier [2007] und Maier und Möller [2007] beschreiben Tücken von Metaanalysen aus der Psychopharmakaforschung und betonen in zwei verdienstvollen Veröffentlichungen die Wichtigkeit, zu einer international einheitlichen Evidenzgraduierung zu kommen. Diese muss auch die Besonderheiten von Psychotherapiestudien mit einschließen, bei denen Doppelblindheit nicht möglich und randomisierte Studien nicht die einzige Form gut kontrollierter Studien sind. Besonders bemerkenswert ist, dass so gut wie alle nationalen und internationalen Zulassungsbehörden für Arzneimittel ihre Zulassungsentscheidungen nicht auf der Basis von Metaanalysen,

\begin{tabular}{|c|c|}
\hline KARGER & (c) 2008 S. Karger GmbH, Freiburg \\
\hline $\begin{array}{l}\text { Fax +497614520714 } \\
\text { E-mail Information@Karger.de } \\
\text { www.karger.com }\end{array}$ & $\begin{array}{l}\text { Accessible online at: } \\
\text { www.karger.com/ver }\end{array}$ \\
\hline
\end{tabular}


sondern auf der Basis von qualifizierten oder systematischen Reviews und sogar auf der Basis gut belegter Einzelstudien fällen.

\section{Probleme und Grenzen von Metaanalysen}

1. Folgerungen aus Metaanalysen sind retrospektiv. In der Regel werden Studien aus einem längeren Zeitraum zusammengefasst, um überhaupt zu Aussagen kommen zu können. Metaanalysen resümieren somit die Ergebnisse von «vorgestern». Auch sind Metaanalysen nicht wirklich Metaanalysen! Es gehen in den Datensatz einer Metaanalyse nicht die ursprünglichen Daten der eingeschlossenen Studien ein, sondern lediglich deren Mittelwerte und Streuungen. Aus diesen werden dann die Effektstärken und die Konfidenzintervalle berechnet.

2. Melander et al. [2003] berichteten in ihrem Artikel «Evidence B(i)ased Medicine» über Auswirkungen von «Publikations-Bias» auf die Ergebnisse von Metaanalysen. Die meisten Metaanalysen schließen nur veröffentlichte Studienergebnisse ein. So verfährt beispielsweise die Cochrane Collaboration bei ihren Metaanalysen. Hinsichtlich möglicher Auswirkungen von selektiven SerotoninWiederaufnahmehemmern (SSRIs) auf die Anzahl von Suizidversuchen im Vergleich zu Placebo kommt man jedoch zu unterschiedlichen Ergebnisse - je nachdem, auf welcher Stichprobenbasis die Metaanalysen durchgeführt werden: Die Metaanalyse von Gunnell et al. [2005] basierte auf allen placebokontrollierten Studien, die in der Datenbank von Medline bzw. im Cochrane-Register gelistet waren. Eine Metaanalyse von Fergusson et al. [2005] basierte dagegen auf allen Studien zu diesem Thema, die bei der Registrierbehörde «Medicines and Healthcare Production Regulation Agency» gemeldet waren. Nach den Ergebnissen der Metaanalyse von Gunnell et al. fand sich unter SSRIs keine höhere Suizidzahl als unter Placebo. Dagegen fand sich ein Unterschied zuungunsten der SSRIs in der Metaanalyse von Fergusson et al. Es spielt also eine wesentliche Rolle, welche Datenbasis zugrunde gelegt wird.

3. In Bereichen, zu denen sehr viele empirische Studien vorliegen, kann es bei einer Zusammenführung aller Einzelstudien zu einem so großen Stichprobenumfang kommen, dass die Ergebnisse als «überpowert» anzusehen sind. Das bedeutet, dass auch geringfügige, klinisch nicht relevante Faktoren eine Signifikanz aufweisen können. Umgekehrt kommt es in Bereichen, zu denen nur wenige in Design und Ergebnissen heterogene Studien vorliegen, zu einer «Unterpowerung». Aussagen, die bei genauer Betrachtung gut geplanter einzelner Studien sehr klar erscheinen, finden sich dann in den in diesem Fall nichtssagenden zusammenfassenden Statements einer solchen Metaanalyse wieder. Dies ist der Fall beispielsweise bei der Cochrane Metaanalyse zur ambulanten Einzelpsychotherapie bei Er- wachsenen mit Anorexia nervosa [Hay et al., 2004]: «No specific treatment was consistently superior to any other specific approach», heißt es da lapidar in der Zusammenfassung.

4. Metaanalysen gehen in der Regel davon aus, dass die ausgewählten Studien für die Testung der Prüfhypothese direkt vergleichbar sind. Die dafür erforderliche Homogenität der Effektstärken der eingeschlossenen Studien ist jedoch meistens nicht gegeben. Die Inhomogenität der Effektstärken der eingeschlossenen Studien wird hier als unerwünscht und störend gesehen. Eine genauere Betrachtung dieser Varianz kann allerdings auch sehr informativ sein. Dies wird in Metaanalysen aber nicht berücksichtigt. Ein Beispiel dafür, dass diese Varianz von Interesse ist, ist die Metaanalyse von Anderson [2000] über die antidepressive Wirksamkeit von SSRIs im Vergleich zu trizyklischen Antidepressiva. Aus den Ergebnissen der ersten Metaanalyse folgerte Anderson, dass zwar die Befundlage inkonsistent war, trizyklische Antidepressiva jedoch etwas besser abschnitten. Einige Jahre später wurde das Datenmaterial unter Berücksichtigung verschiedener Behandlungssettings reanalysiert [Anderson, 2007]. In dieser differenzierteren, späteren Metaanalyse waren die Ergebnisse eindeutiger: Sie zeigten eine bessere Wirksamkeit von trizyklischen Antidepressiva im stationären und von SSRIs im ambulanten Setting.

5. Unterschiedliche Ergebnisse von Metaanalysen durch unterschiedliche Teststatistiken: Im Idealfall sollten alle vorliegenden Studien zu einem Thema in eine Metaanalyse eingeschlossen werden. Aus vernünftigen Gründen werden in der Praxis allerdings Definitionen erarbeitet, die einzelne Studien ausschließen, teils weil sie keine ausreichend fundierte empirische Qualität haben, teils weil sie vom Design her nicht ganz hineinpassen mögen. Wenn bei diesem Schritt eine wichtige Studie z.B. aus Designgründen ausgeschlossen wird, kann dies die Ergebnisse erheblich verzerren und beachtliche Veränderungen nach sich ziehen. Ein Beispiel ist der Ausschluss wichtiger Studien bei der Cochrane Metaanalyse «Antidepressiva versus Psychotherapie und ihre Kombination bei Bulimia nervosa». Von 35 veröffentlichten empirischen Studien zu dem Thema wurden nur 7 in die Metaanalyse eingeschlossen.

Metaanalysen sind nur eines von verschiedenen Hilfsmitteln für die Ableitung von Hypothesendaten. Sie eignen sich jedoch nicht besonders als Methode der Evidenzmaximierung für Therapieempfehlungen, z.B. in empirisch fundierten S3-Leitlinien. Die Formulierung von Leitlinien, die 10 oder mehr Jahre zurückgehen, war geprägt von der Bewertung einzelner wichtiger Studien, der Bewertung von qualifizierten oder systematischen Reviews sowie insbesondere auch der Expertenmeinung (S1- und S2-Leitlinien). Unter S3-Leitlinien versteht man solche, die empirisch fundierte Evidenzen aufzeigen, diese nach Evidenzgraden bewerten und konkrete Empfehlungen aussprechen. Leitlinien sind rückwärts gerichtet, führen somit zu eher konservativen (gegebenenfalls veralteten) Therapieent- 
scheidungen und tragen bei einem sich schnell entwickelnden Feld neuen Erkenntnissen nicht genügend Rechnung. Auch wenn von vielen Seiten betont wird, Leitlinien seien für den Behandelnden nicht rechtlich verpflichtend, Leitlinien seien keine Richtlinien, muss dennoch davon ausgegangen werden, dass Gesundheitsbehörden und Krankenkassen diese als Argument für Einsparungen im Gesundheitswesen nehmen werden. Es erscheint zwar legitim, dass der Staat oder die Krankenkassen nur Behandlungen finanzieren, deren Wirksamkeit nachgewiesen und gegebenenfalls anderen Behandlungsmethoden überlegen ist. Die obige Diskussion zu Metaanalysen zeigt allerdings, welche Vorsicht geboten ist. Fehlschlüsse oder gar Missbrauch können hier nicht ausgeschlossen werden. Bei unkritischer Anwendung von Metaanalysen und unkritischen Bewertungen in S3-Leitlinien kann es auch zu gesundheitspolitischen Fehlentwicklungen kommen, die Auswirkungen auf Ihre psychotherapeutische Tätigkeit haben können.

Eine der Arbeiten in diesem Heft [Böhm et al., 2008] befasst sich unmittelbar mit Leitlinien, die bei Zwangsstörungen eindeutig Expositionsverfahren empfehlen. Die Autoren kommen aufgrund ihrer empirischen Daten zu dem Schluss, dass die Leitlinienempfehlungen in diesem Bereich kaum beachtet werden. Auch bei drei weiteren Arbeiten in diesem Heft handelt es sich um empirische Originalarbeiten. Gerhards [2008] berichtet in einem RCT über die Effekte von Ablenkungsund Entspannungstraining bei chronischem Tinnitus, Runge et al. [2008] über die Epidemiologie von Angststörungen bei einer repräsentativen Bevölkerungserhebung bei 14- bis 34Jährigen (EDSP) und die Inanspruchnahme von Psychotherapie und Linden et al. [2008] berichten über die Entwicklung eines Fragebogens zum Therapieerleben von Patienten und Angehörigen. Die kurze Arbeit von Isensee et al. [2008] in diesem Heft stellt ein ambulantes verhaltensmedizinisches Behandlungsmodell für chronisch kranke Kinder und Jugendliche vor. Der Höhepunkt des Heftes ist ein Interview, das Dr. Gaby Bleichhardt mit Prof. Dr. Paul Salkovskis (London) führte. Er ist einer der bedeutendsten Verhaltenstherapeuten der Gegenwart. Seine Hauptforschungsgebiete sind «Health Anxieties», Hypochondrie und kognitive Verhaltenstherapie. Am Rande sei erwähnt, dass er auch internationaler Beirat von Verhaltenstherapie ist. Die Lektüre der Arbeiten in diesem Heft ist wirklich lohnend.

\section{Literatur}

Anderson IM: Selective serotonin reuptake inhibitors versus tricyclic antidepressants: a meta-analysis of efficacy and tolerability. J Affect Disord 2000;58:19-36. Anderson IM: SSRIs versus tricyclic antidepressants in depressed inpatients: a meta-analysis of efficacy and tolerability. Depress Anxiety 2007;7(suppl 1):11-17. Berger M, Antes G, Hecht H: Die Lehrbuchrelevanz von evidenzbasierter Medizin und der Cochrane Collaboration; in BergerM (Hrsg): Psychische Erkrankungen - Klinik und Therapie, ed 2. München, Urban und Fischer, 2004, pp 3-16.

Böhm K, Förster U, Külz A, Voderholzer U: Versorgungsrealität der Zwangsstörungen: Werden Expositionsverfahren eingesetzt? Verhaltenstherapie 2008;18: $18-24$

Fergusson D, Doucette S, Glass KC, et al: Association between suicide attempts and selective serotonin reuptake inhibitors: systematic review of randomised controlled trials. Br Med J 2005;330:396-402.
Gerhards F: Effekte von Ablenkungs- und Entspannungstraining bei chronischem Tinnitus und Bedeutung der individuellen Fallkomplexität. Verhaltenstherapie 2008;18:9-17.

Gunnell D, Saperia J, Ashby D: Selective serotonin reuptake inhibitors (SSRIs) and suicide in adults: meta-analysis of drug company data from placebo controlled, randomised controlled trials submitted to the MHRA's safety review. Br Med J 2005;330:385-389. Hay P, Bacaltchuk J, Claudino a, Ben-Tovim D, Yong PY: Individual psychotherapy in the outpatient treatment of adults with anorexia nervosa. The Cochrane Library. Issue 3. Chichester, Wiley, 2004, www.cochrane. org.

Isensee B, Gerber-von Müller G, Kropp P; Stephani U, Gerber WD: Ein ambulantes verhaltensmedizinisches Behandlungsmodell für chronisch kranke Kinder und Jugendliche. Konzeption und Erfahrungen in der Praxis. Verhaltenstherapie 2008;18:43-48.

Linden M, Lind A, Quosh C: Der TherapieerlebenFragebogen (TeR) für Patienten und Angehörige. Verhaltenstherapie 2008;18:35-42.
Maier W, Möller H-J: Metaanalysen. Methode zur Evidenzmaximierung von Therapiestudien? Nervenarzt 2007:78:1028-1036.

Melander H, Ahlqvist-Rastad J, Meijer G, Beermann B: Evidence b(i)ased medicine - selective reporting from studies sponsored by pharmaceutical industry: review of studies in new drug applications. Br Med J 2003:326:1171-1173.

Möller H-J, Maier W: Probleme der «evidence-based medicine» in der Psychopharmakotherapie. Problematik der Evidenzgraduierung und der Evidenzbasierung komplexer klinischer Entscheidungsprozesse. Nervenarzt 2007;78:1014-1027.

Runge AJ, Beesdo K, Lieb R, Wittchen HU: Wie häufig nehmen Jugendliche und junge Erwachsene mit Angststörungen eine psychotherapeutische Behandlung in Anspruch? Verhaltenstherapie 2008;18:26-34. Salkovskis P: Die kognitive Verhaltenstherapie ist keine perfekte Therapie, nur die beste, die wir zurzeit haben. Verhaltenstherapie 2008;18:49-52. 